\title{
Well-Posedness for a Class of Strongly Mixed Variational-Hemivariational Inequalities with Perturbations
}

\author{
Lu-Chuan Ceng, ${ }^{1,2}$ Ngai-Ching Wong, ${ }^{3}$ and Jen-Chih Yao ${ }^{4}$ \\ ${ }^{1}$ Department of Mathematics, Shanghai Normal University, Shanghai 200234, China \\ ${ }^{2}$ Scientific Computing Key Laboratory, Shanghai Universities, Shanghai 200234, China \\ ${ }^{3}$ Department of Applied Mathematics, National Sun Yat-Sen University, Kaohsiung 80424, Taiwan \\ ${ }^{4}$ Center for General Education, Kaohsiung Medical University, Kaohsiung 80708, Taiwan
}

Correspondence should be addressed to Ngai-Ching Wong, wong@math.nsysu.edu.tw

Received 1 August 2011; Accepted 19 November 2011

Academic Editor: Ya Ping Fang

Copyright ( $) 2012$ Lu-Chuan Ceng et al. This is an open access article distributed under the Creative Commons Attribution License, which permits unrestricted use, distribution, and reproduction in any medium, provided the original work is properly cited.

\begin{abstract}
The concept of well-posedness for a minimization problem is extended to develop the concept of well-posedness for a class of strongly mixed variational-hemivariational inequalities with perturbations which includes as a special case the class of variational-hemivariational inequalities with perturbations. We establish some metric characterizations for the well-posed strongly mixed variational-hemivariational inequality and give some conditions under which the strongly mixed variational-hemivariational inequality is strongly well-posed in the generalized sense. On the other hand, it is also proven that under some mild conditions there holds the equivalence between the well posedness for a strongly mixed variational-hemivariational inequality and the well-posedness for the corresponding inclusion problem.
\end{abstract}

\section{Introduction}

It is well known that the classical notion of well-posedness for the minimization problem (MP) is due to Tykhonov [1], which has been known as the Tykhonov well-posedness. Let $V$ be a Banach space and $f: V \rightarrow \mathbf{R} \cup\{+\infty\}$ be a real-valued functional on $V$. The problem $(\mathrm{MP})$, that is, $\min _{x \in V} f(x)$, is said to be well posed if there exists a unique minimizer and every minimizing sequence converges to the unique minimizer. Furthermore, the notion of generalized Tykhonov well-posedness is also introduced for the problem (MP), which means the existence of minimizers and the convergence of some subsequence of every minimizing sequence toward a minimizer. Clearly, the concept of well-posedness is inspired by numerical methods producing optimizing sequences for optimization problems and plays a crucial 
role in the optimization theory. Therefore, various concepts of well-posedness have been introduced and studied for optimization problems. For more details, we refer to [2-8] and the references therein.

On the other hand, the concept of well-posedness has been extended to other related problems, such as variational inequalities [5, 9-14], saddle-point problem [15], inclusion problems [10,11], and fixed-point problems [10,11]. An initial notion of well-posedness for variational inequalities is due to Lucchetti and Patrone [5]. They introduced the notion of well-posedness for variational inequalities and proved some related results by means of Ekeland's variational principle. Since then, many authors have been devoted to generating the concept of well-posedness from the minimization problem to various variational inequalities. In [2], Crespi et al. gave the notions of well-posedness for a vector optimization problem and a vector variational inequality of the differential type, explored their basic properties, and investigated their links. Lignola [13] introduced two concepts of well-posedness and $L$-well-posedness for quasivariational inequalities and investigated some equivalent characterizations of these two concepts. Recently, Fang et al. [11] generalized the concepts of well-posedness and $\alpha$-well-posedness to a generalized mixed variational inequality which includes as a special case the classical variational inequality and discussed its links with the well-posedness of corresponding inclusion problem and the well-posedness of corresponding fixed-point problem. They also derived some conditions under which the mixed variational inequality is well posed. For further results on the well-posedness for variational inequalities and equilibrium problems, we refer to $[5,8,11,13,16-18]$ and the references therein.

In 1983, in order to formulate variational principles involving energy functions with no convexity and no smoothness, Panagiotopoulos [19] first introduced the hemivariational inequality which is an important and useful generalization of variational inequality and investigated it by using the mathematical notion of the generalized gradient of Clarke for nonconvex and nondifferentiable functions [20]. The hemivariational inequalities have been proved very efficient to describe a variety of mechanical problems, for instance, unilateral contact problems in nonlinear elasticity, problems describing the adhesive and frictional effects, and nonconvex semipermeability problems (see, for instance, [19, 21, 22]). Therefore, in recent years all kinds of hemivariational inequalities have been studied by many authors [14, 21, 23-29], and the study of hemivariational inequalities has emerged as a new and interesting branch of applied mathematics. However, there are very few researchers extending the well-posedness to hemivariational inequalities. In 1995, Goeleven and Mentagui [14] first introduced the notion of well-posedness for hemivariational inequalities and established some basic results concerning the well-posed hemivariational inequality.

Very recently, Xiao and Huang [30] generalized the well-posedness of minimization problems to a class of variational-hemivariational inequalities with perturbations, which includes as special cases the classical hemivariational inequalities and variational inequalities. Under appropriate conditions, they derived some metric characterizations for the wellposed variational-hemivariational inequality and presented some conditions under which the variational-hemivariational inequality is strongly well posed in the generalized sense. Meantime, they also proved that the well-posedness for a variational-hemivariational inequality is equivalent to the well-posedness for the corresponding inclusion problem.

In this paper, we extend the notion of well-posedness for minimization problems to a class of strongly mixed variational-hemivariational inequalities with perturbations, which includes as a special case the class of variational-hemivariational inequalities with perturbations in [30]. Under very mild conditions, we establish some metric characterizations for the well-posed strongly mixed variational-hemivariational inequality and give some conditions 
under which the strongly mixed variational-hemivariational inequality is strongly wellposed in the generalized sense. On the other hand, it is also proven that the well-posed- ness for a strongly mixed variational-hemivariational inequality is equivalent to the well-posedness for the corresponding inclusion problem.

\section{Preliminaries}

Throughout this paper, unless stated otherwise, we always suppose that $V$ is a real reflexive Banach space, where its dual space is denoted by $V^{*}$ and the generalized duality pairing between $V$ and $V^{*}$ is denoted by $\langle\cdot, \cdot\rangle$. We denote the norms of Banach spaces $V$ and $V^{*}$ by $\|\cdot\|_{V}$ and $\|\cdot\|_{V^{*}}$, respectively. In what follows, let $N: V^{*} \times V^{*} \rightarrow V^{*}, A, T: V \rightarrow V^{*}$ and $g: V \rightarrow V$ be four mappings, $G: V \rightarrow \mathbf{R} \cup\{+\infty\}$ be a proper, convex, and lower semicontinuous functional, and $f \in V^{*}$ be some given element. Denote by dom $G$ the efficient domain of functional, that is,

$$
\operatorname{dom} G:=\{u \in V: G(u)<+\infty\}
$$

Consider the following strongly mixed variational-hemivariational inequality: find $u \in$ $V$ such that

SMVHVI : $\langle N(A g(u), T u)-f, v-g(u)\rangle+J^{\circ}(u, v-g(u))+G(v)-G(g(u)) \geq 0, \quad \forall v \in V$,

where $J^{\circ}(u, v)$ denotes the generalized directional derivative in the sense of Clarke of a locally Lipschitz functional $J: V \rightarrow \mathbf{R}$ at $u$ in the direction $v$ (see [20]) given by

$$
J^{\circ}(u, v):=\limsup _{w \rightarrow u \lambda \downarrow 0} \frac{J(w+\lambda v)-J(w)}{\lambda} .
$$

In particular, if $N\left(u^{*}, v^{*}\right)=u^{*}+v^{*}$, for all $u^{*}, v^{*} \in V^{*}$ and $g=I$ the identity mapping of $V$, then the problem (2.2) reduces to the following variational-hemivariational inequality of finding $u \in V$ such that

$$
\text { VHVI : } \quad\langle A u+T u, v-u\rangle+J^{\circ}(u, v-u)+G(v)-G(u) \geq\langle f, v-u\rangle, \quad \forall v \in V,
$$

where $T$ is perturbation, which was first introduced and studied by Xiao and Huang [30].

Let $\Omega$ be an open bounded subset of $\mathrm{R}^{3}$ which is occupied by a linear elastic body and $\Gamma$ the boundary of the $\Omega$ which is assumed to be appropriately regular $\left(C^{0,1}\right.$, i.e., a Lipschitzian boundary, is sufficient). We denote by $S=\left\{S_{i}\right\}$ the stress vector on $\Gamma$, which can be decomposed into a normal component $S_{N}$ and a tangential component $S_{T}$ with respect to $\Gamma$, that is,

$$
S_{N}=\sigma_{i j} n_{j} n_{i}, \quad S_{T_{i}}=\sigma_{i j} n_{j}-\left(\sigma_{i j} n_{i} n_{j}\right) n_{i}
$$

where $\sigma=\left\{\sigma_{i j}\right\}$ is an appropriately defined stress tensor and $n=\left\{n_{i}\right\}$ is the outward unit normal vector on $\Gamma$. Analogously, $u_{N}$ and $u_{T}$ denote the normal and the tangential 
components of the displacement vector $u$ with respect to $\Gamma$. As pointed out in [30], the reaction-displacement law presents in compression ideal locking effect (the infinite branch $E F$ ), that is, always $u_{N} \leq a$, whereas $u_{N}>a$ is impossible. Specifically,

$$
\begin{aligned}
& \text { if } u_{N}<a \text { then }-S_{N} \in \tilde{\beta}\left(u_{N}\right), \\
& \text { if } u_{N}=a \text { then }-\infty<-S_{N} \leq \tilde{\beta}(a), \\
& \text { if } u_{N}>a \text { then } S_{N}=\emptyset,
\end{aligned}
$$

where $\tilde{\beta}$ is a multivalued function defined as follows. Suppose that $\beta: \mathbf{R} \rightarrow \mathbf{R}$ is a function such that $\beta \in L_{\text {loc }}^{\infty}(\mathbf{R})$, that is, a function essentially bounded on any bounded interval of $\mathbf{R}$. For any $\rho>0$ and $\xi \in \mathbf{R}$, we define $\bar{\beta}_{\rho}(\xi)=\operatorname{ess} \inf _{\left|\xi_{1}-\xi\right| \leq \rho} \beta\left(\xi_{1}\right)$ and $\overline{\bar{\beta}}_{\rho}(\xi)=\operatorname{ess}_{\sup _{\mid \xi_{1}-\xi} \mid \leq \rho} \beta\left(\xi_{1}\right)$. By the monotonicity of the functions $\bar{\beta}_{\rho}$ and $\overline{\bar{\beta}}_{\rho}$ with respect to $\rho$, we infer that the limits as $\rho \rightarrow 0_{+}$exist, that is,

$$
\bar{\beta}(\xi)=\lim _{\rho \rightarrow 0_{+}} \bar{\beta}_{\rho}(\xi), \quad \overline{\bar{\beta}}(\xi)=\lim _{\rho \rightarrow 0_{+}} \overline{\bar{\beta}}_{\rho}(\xi)
$$

Then,

$$
\tilde{\beta}(\xi)=[\bar{\beta}(\xi), \overline{\bar{\beta}}(\xi)]
$$

Furthermore, a locally Lipschitz function $j_{N}$ can be determined up to an additive constant by

$$
j_{N}(\xi)=\int_{0}^{\xi} \beta\left(\xi_{1}\right) d \xi_{1}
$$

such that $\bar{\partial} j_{N}(\xi)=\tilde{\beta}(\xi)$ for each $\xi \in \mathbf{R}$ when the limits $\beta\left(\xi_{ \pm}\right)$exist, where $\bar{\partial} j_{N}$ is the Clarke's generalized gradient of locally Lipschitz function $j_{N}$ which will be specified in what follows.

Now, let $K=\left\{u_{N} \mid u_{N} \leq a\right\}, N_{K}$ the normal cone to $K$ at $u_{N}$, and $I_{K}$ the indicator of the set $K$. Then (2.6) can be written as

$$
-S_{N} \in \tilde{\beta}\left(u_{N}\right)+N_{K}\left(u_{N}\right)=\bar{\partial} j_{N}\left(u_{N}\right)+\partial I_{K}\left(u_{N}\right),
$$

where $\partial I_{K}$ is the subgradient of the convex functional $I_{K}$ in the sense of convex analysis, which will also be specified in what follows. By the definitions of the Clarke's generalized gradient of locally Lipschitz function and the subgradient of the convex functional, (2.10) gives rise to the following variational-hemivariational inequality

$$
u_{N} \in \mathbf{R}: \quad\left\langle S_{N}, v-u_{N}\right\rangle+j_{N}^{\circ}\left(u_{N}, v-u_{N}\right)+I_{K}(v)-I_{K}\left(u_{N}\right) \geq 0, \quad \forall v \in \mathbf{R},
$$

which is a special case of the variational-hemivariational inequality VHVI. Beyond question, the problem (2.11) is a special case of the strongly mixed variational-hemivariational inequality SMVHVI as well. More special cases of the SMVHVI are stated as follows. 
(i) If $G=\delta_{K}$ and $J(u)=\int_{\Omega} j(x, u) d \Omega$, where $\delta_{K}$ denotes the indicator functional of a nonempty, convex subset $K$ of a function space $V$ defined on $\Omega$ and $j: \Omega \times \mathbf{R} \rightarrow \mathbf{R}$ is a locally Lipschitz continuous function, then the SMVHVI reduces to the following strongly mixed variational-hemivariational inequality:

SMVHVI : $\quad\langle N(A g(u), T u)-f, v-g(u)\rangle+J^{\circ}(u, v-g(u)) \geq 0, \quad \forall v \in K$.

Remark that the SMVHVI (2.12) with $N(A g(u), T u)=A g(u)+T u$ and $g=I$ is equivalent to the VHVI which was considered by Goeleven and Mentagui in [14].

(ii) If $G=0$, then the SMVHVI (2.2) with $N(A g(u), T u)=A g(u)+T u$ reduces to the strongly mixed hemivariational inequality of finding $u \in V$ such that

SMHVI : $\quad\langle A g(u)+T u-f, v-g(u)\rangle+J^{\circ}(u, v-g(u)) \geq 0, \quad \forall v \in V$.

Remark that the SMHVI (2.13) with $T=0$ and $g=I$ is equivalent to the hemivariational inequality (HVI) studied intensively by many authors (see, e.g., [21, 22]).

(iii) If $J=0$, then the SMVHVI (2.2) with $N(A g(u), T u)=A g(u)+T u$ reduces to the strongly mixed variational inequality of finding $u \in V$ such that

SMVI : $\quad\langle A g(u)+T u-f, v-g(u)\rangle+G(v)-G(g(u)) \geq 0, \quad \forall v \in V$.

Remark that the SMVI (2.14) with $T=0$ and $g=I$ is equivalent to the mixed variational inequality (see, e.g., $[11,31])$ and the references therein).

(iv) If $T=0, J=0, g=I$ and $G=\delta_{K}$, then the SMVHVI (2.2) with $N(A g(u), T u)=$ $A g(u)+T u$ reduces to the classical variational inequality:

$$
\mathrm{VI}: \quad\langle A u-f, v-u\rangle \geq 0, \quad \forall v \in K .
$$

(v) If $N=0, J=0, g=I$, and $f=0$, then the SMVHVI (2.2) reduces to the global minimization problem:

$$
\mathrm{MP}: \quad \min _{u \in V} G(u)
$$

Let $\partial G(u): V \rightarrow 2^{V^{*}} \backslash\{\emptyset\}$ and $\bar{\partial} J(u): V \rightarrow 2^{V^{*}} \backslash\{\emptyset\}$ denote the subgradient of convex functional $G$ in the sense of convex analysis (see [32]) and the Clarke's generalized gradient of locally Lipschitz functional $J$ (see [20]), respectively. That is,

$$
\begin{gathered}
\partial G(u)=\left\{u^{*} \in V^{*}: G(v)-G(u) \geq\left\langle u^{*}, v-u\right\rangle, \forall v \in V\right\}, \\
\bar{\partial} J(u)=\left\{\omega \in V^{*}: J^{\circ}(u, v) \geq\langle\omega, v\rangle, \forall v \in V\right\} .
\end{gathered}
$$


Remark 2.1 (see [33]). The Clarke's generalized gradient of a locally Lipschitz functional $J$ : $V \rightarrow \mathbf{R}$ at a point $u$ is given by

$$
\bar{\partial} J(u)=\partial\left(J^{\circ}(u, \cdot)\right)(0)
$$

About the subgradient in the sense of convex analysis, the Clarke's generalized directional derivative, and the Clarke's generalized gradient, we have the following basic properties (see, e.g., $[20,30,32,33])$.

Proposition 2.2. Let $V$ be a Banach space and $G: V \rightarrow \mathbf{R} \cup\{+\infty\}$ be a convex and proper functional. Then we have the following properties of $\partial G$ :

(i) $\partial G(u)$ is convex and weak ${ }^{*}$-closed;

(ii) if $G$ is continuous at $u \in \operatorname{dom} G$, then $\partial G(u)$ is nonempty, convex, bounded, and weak ${ }^{*}$ compact;

(iii) if $G$ is Gateaux differentiable at $u \in \operatorname{dom} G$, then $\partial G(u)=\{D G(u)\}$, where $D G(u)$ is the Gateaux derivative of $G$ at $u$.

Proposition 2.3. Let $V$ be a Banach space and $G_{1}, G_{2}: V \rightarrow \mathbf{R} \cup\{+\infty\}$ be two convex functionals. If there is a point $u_{0} \in \operatorname{dom} G_{1} \cap \operatorname{dom} G_{2}$ at which $G_{1}$ is continuous, then the following equation holds:

$$
\partial\left(G_{1}+G_{2}\right)(u)=\partial G_{1}(u)+\partial G_{2}(u), \quad \forall u \in V
$$

Proposition 2.4. Let $V$ be a Banach space, $u, v \in V$, and $J$ a locally Lipschitz functional defined on $V$. Then

(i) the function $v \mapsto J^{\circ}(u, v)$ is finite, positively homogeneous, subadditive, and then convex on $\mathrm{V}$;

(ii) $J^{\circ}(u, v)$ is upper semicontinuous as a function of $(u, v)$, as a function of $v$ alone, is Lipschitz continuous on $V$;

(iii) $J^{\circ}(u-v)=(-J)^{\circ}(u, v)$;

(iv) $\bar{\partial} J(u)$ is a nonempty, convex, bounded, weak ${ }^{*}$-compact subset of $V^{*}$;

(v) for every $v \in V$, one has

$$
J^{\circ}(u, v)=\max \{\langle\xi, v\rangle: \xi \in \bar{\partial} J(u)\}
$$

Now we recall some important definitions and useful results.

Definition 2.5 (see [34]). Let $V$ be a real Banach space with its dual $V^{*}$ and $T$ be an operator from $V$ to its dual space $V^{*}$. $T$ is said to be monotone if

$$
\langle T u-T v, u-v\rangle \geq 0, \quad \forall u, v \in V .
$$


Definition 2.6 (see [34]). A mapping $T: V \rightarrow V^{*}$ is said to be hemicontinuous if for any $u, v \in V$, the function $t \mapsto\langle T(u+t(v-u)), v-u\rangle$ from $[0,1]$ into $\mathbf{R}$ is continuous at $0_{+}$.

It is clear that the continuity implies the hemicontinuity, but the converse is not true in general.

Theorem 2.7 (see [35]). Let $C \subset V$ be nonempty, closed, and convex, $C^{*} \subset V^{*}$ nonempty, closed, convex, and bounded, $\varphi: V \rightarrow \mathbf{R} \cup\{+\infty\}$ proper, convex, and lower semicontinuous, and $y \in C$ be arbitrary. Assume that, for each $x \in C$, there exists $x^{*}(x) \in C^{*}$ such that

$$
\left\langle x^{*}(x), x-y\right\rangle \geq \varphi(y)-\varphi(x)
$$

Then, there exists $y^{*} \in C^{*}$ such that

$$
\left\langle y^{*}, x-y\right\rangle \geq \varphi(y)-\varphi(x), \quad \forall x \in C
$$

Definition 2.8 (see [36]). Let $S$ be a nonempty subset of $V$. The measure, say $\mu$, of noncompactness for the set $S$ is defined by

$$
\mu(S):=\inf \left\{\epsilon>0: S \subset \bigcup_{i=1}^{n} S_{i}, \operatorname{diam} S_{i}<\epsilon, i=1,2, \ldots, n\right\},
$$

where diam $S_{i}$ means the diameter of set $S_{i}$.

Definition 2.9 (see [36]). Let $A, B$ be nonempty subsets of $V$. The Hausdorff metric $\mathscr{H}(\cdot, \cdot)$ between $A$ and $B$ is defined by

$$
\mathscr{H}(A, B):=\max \{e(A, B), e(B, A)\}
$$

where $e(A, B):=\sup _{a \in A} d(a, B)$ with $d(a, B):=\inf _{b \in B}\|a-b\|_{V}$.

Let $\left\{A_{n}\right\}$ be a sequence of nonempty subsets of $V$. We say that $A_{n}$ converges to $A$ in the sense of Hausdorff metric if $\mathscr{L}\left(A_{n}, A\right) \rightarrow 0$. It is easy to see that $e\left(A_{n}, A\right) \rightarrow 0$ if and only if $d\left(a_{n}, A\right) \rightarrow 0$ for all section $a_{n} \in A_{n}$. For more details on this topic, we refer the reader to [36].

\section{Well-Posedness of the SMVHVI with Metric Characterizations}

In this section, we generalize the concept of well-posedness to the strongly mixed variational-hemivariational inequality SMVHVI with perturbations, establish its metric characterizations, and derive some conditions under which the strongly mixed variational-hemivariational inequality is strongly well-posed in the generalized sense in Euclidean space $\mathbf{R}^{n}$. 
Definition 3.1. A sequence $\left\{u_{n}\right\} \subset V$ is said to be an approximating sequence for the SMVHVI if there exists a nonnegative sequence $\left\{\epsilon_{n}\right\}$ with $\epsilon_{n} \rightarrow 0$ as $n \rightarrow \infty$ such that

$$
\begin{aligned}
& \left\langle N\left(A g\left(u_{n}\right), T u_{n}\right)-f, v-g\left(u_{n}\right)\right\rangle+J^{\circ}\left(u_{n}, v-g\left(u_{n}\right)\right)+G(v)-G\left(g\left(u_{n}\right)\right) \\
& \quad \geq-\epsilon_{n}\left\|v-g\left(u_{n}\right)\right\|_{V}, \quad \forall v \in V .
\end{aligned}
$$

Definition 3.2. The SMVHVI is said to be strongly (resp., weakly) well posed if the SMVHVI has a unique solution in $V$ and every approximating sequence converges strongly (resp., weakly) to the unique solution.

Remark 3.3. Strong well-posedness implies weak well-posedness, but the converse is not true in general.

Definition 3.4. The SMVHVI is said to be strongly (resp., weakly) well posed in the generalized sense if the SMVHVI has a nonempty solution set $S$ in $V$ and every approximating sequence has a subsequence which converges strongly (resp., weakly) to some point of the solution set $S$.

Remark 3.5. Strong well-posedness in the generalized sense implies weak well-posedness in the generalized sense, but the converse is not true in general.

Definition 3.6. Let $N: V^{*} \times V^{*} \rightarrow V^{*}$ and $A: V \rightarrow V^{*}$ be two mappings. Then

(i) $A$ is said to be monotone with respect to the first argument of $N$ if there holds

$$
\left\langle N\left(A u, w^{*}\right)-N\left(A v, w^{*}\right), u-v\right\rangle \geq 0, \quad \forall u, v \in V, w^{*} \in V^{*}
$$

(ii) $A$ is said to be continuous with respect to the first argument of $N$ if for each $w^{*} \in V^{*}$ the mapping $v \mapsto N\left(A v, w^{*}\right)$ from $V$ into $V^{*}$ is continuous;

(iii) $A$ is said to be hemicontinuous with respect to the first argument of $N$ if for all $u, v \in V$ and $w^{*} \in V^{*}$, the function $t \mapsto\left\langle N\left(A(u+t(v-u)), w^{*}\right), v-u\right\rangle$ from $[0,1]$ into $\mathbf{R}$ is continuous at $0_{+}$.

For any $\epsilon>0$, we define the following two sets:

$$
\begin{aligned}
\Omega(\epsilon) & =\left\{u \in V:\langle N(A g(u), T u)-f, v-g(u)\rangle+J^{\circ}(u, v-g(u))+G(v)-G(g(u))\right. \\
& \left.\geq-\epsilon\|v-g(u)\|_{V}, \forall v \in V\right\}, \\
\Psi(\epsilon) & =\left\{u \in V:\langle N(A v, T u)-f, v-g(u)\rangle+J^{\circ}(u, v-g(u))+G(v)-G(g(u))\right. \\
& \left.\geq-\epsilon\|v-g(u)\|_{V}, \forall v \in V\right\} .
\end{aligned}
$$

Lemma 3.7. Suppose that $A: V \rightarrow V^{*}$ is both monotone and hemicontinuous with respect to the first argument of $N, G: V \rightarrow \mathbf{R} \cup\{+\infty\}$ is a proper, convex, and lower semicontinuous functional. Then $\Omega(\epsilon)=\Psi(\epsilon)$ for all $\epsilon>0$. 
Proof. Let $u \in \Omega(\epsilon)$. Then, by the monotonicity of the mapping $A$ with respect to the first argument of $N$, we have for all $v \in V$

$$
\begin{aligned}
0 & \leq\langle N(A g(u), T u)-f, v-g(u)\rangle+J^{\circ}(u, v-g(u))+G(v)-G(g(u))+\epsilon\|v-g(u)\|_{V} \\
& \leq\langle N(A v, T u)-f, v-g(u)\rangle+J^{\circ}(u, v-g(u))+G(v)-G(g(u))+\epsilon\|v-g(u)\|_{V} .
\end{aligned}
$$

This implies that $u \in \Psi(\epsilon)$. Thus, we get the inclusion $\Omega(\epsilon) \subset \Psi(\epsilon)$.

Next let us show that $\Psi(\epsilon) \subset \Omega(\epsilon)$. Indeed, for any $u \in \Psi(\epsilon)$, we have

$$
\langle N(A v, T u)-f, v-g(u)\rangle+J^{\circ}(u, v-g(u))+G(v)-G(g(u)) \geq-\epsilon\|v-g(u)\|_{V^{\prime}} \quad \forall v \in V .
$$

For any $w \in V$ and $t \in[0,1]$, putting $v=t w+(1-t) g(u)=g(u)+t(w-g(u))$ in (3.5), we obtain

$$
\begin{aligned}
-\epsilon\|t(w-g(u))\|_{V} \leq & \langle N(A(t w+(1-t) g(u)), T u)-f, t(w-g(u))\rangle \\
& +J^{\circ}(u, t(w-g(u)))+G(t w+(1-t) g(u))-G(g(u)) .
\end{aligned}
$$

Since the Clarke's generalized directional derivative $J^{\circ}(u, v)$ is positively homogeneous with respect to $v$ and $G$ is convex, it follows that

$$
\begin{aligned}
& \langle N(A(t w+(1-t) g(u)), T u)-f, w-g(u)\rangle+J^{\circ}(u, w-g(u))+G(w)-G(g(u)) \\
& \quad \geq-\epsilon\|w-g(u)\|_{V} .
\end{aligned}
$$

Taking the limit for (3.7) as $t \rightarrow 0_{+}$, we obtain from the hemicontinuity of the mapping $A$ with respect to the first argument of $N$ that

$$
\begin{aligned}
& \langle N(A g(u), T u)-f, w-g(u)\rangle+J^{\circ}(u, w-g(u))+G(w)-G(g(u)) \\
& \quad \geq-\epsilon\|w-g(u)\|_{V} .
\end{aligned}
$$

By the arbitrariness of $w \in V$, we conclude that $u \in \Omega(\epsilon)$, which implies that $\Psi(\epsilon) \subset \Omega(\epsilon)$. This completes the proof.

Lemma 3.8. Suppose that $T: V \rightarrow V^{*}$ is continuous with respect to the second argument of $N$, $g: V \rightarrow V$ is continuous, and $G: V \rightarrow \mathbf{R} \cup\{+\infty\}$ is a proper, convex, and lower semicontinuous functional. Then $\Psi(\epsilon)$ is closed in $V$ for all $\epsilon>0$.

Proof. Let $\left\{u_{n}\right\} \subset \Psi(\epsilon)$ be a sequence such that $u_{n} \rightarrow u$ in $V$. Then

$$
\begin{array}{r}
\left\langle N\left(A v, T u_{n}\right)-f, v-g\left(u_{n}\right)\right\rangle+J^{\circ}\left(u_{n}, v-g\left(u_{n}\right)\right)+G(v)-G\left(g\left(u_{n}\right)\right) \geq-\epsilon\left\|v-g\left(u_{n}\right)\right\|_{V^{\prime}} \\
\forall v \in V .
\end{array}
$$


Since $T: V \rightarrow V^{*}$ is continuous with respect to the second argument of $N, g: V \rightarrow V$ is continuous, $G: V \rightarrow \mathbf{R} \cup\{+\infty\}$ is lower semicontinuous, and the Clarke's generalized directional derivative $J^{\circ}(u, v)$ is upper semicontinuous with respect to $(u, v)$, we deduce that $g\left(u_{n}\right) \rightarrow g(u), N\left(A v, T u_{n}\right) \rightarrow N(A v, T u)$, and

$$
\begin{gathered}
\lim _{n \rightarrow \infty}\left\langle N\left(A v, T u_{n}\right), v-g\left(u_{n}\right)\right\rangle=\langle N(A v, T u), v-g(u)\rangle, \\
\limsup _{n \rightarrow \infty} J^{\circ}\left(u_{n}, v-g\left(u_{n}\right)\right) \leq J^{\circ}(u, v-g(u)), \\
\limsup _{n \rightarrow \infty}-G\left(g\left(u_{n}\right)\right) \leq-G(g(u)) .
\end{gathered}
$$

Taking the lim sup for (3.9) as $n \rightarrow \infty$, we obtain from (3.10) that

$$
\langle N(A v, T u)-f, v-g(u)\rangle+J^{\circ}(u, v-g(u))+G(v)-G(g(u)) \geq-\epsilon\|v-g(u)\|_{V^{\prime}} \quad \forall v \in V
$$

which implies that $u \in \Psi(\epsilon)$. Therefore, $\Psi(\epsilon)$ is closed in $V$. This completes the proof.

Corollary 3.9. Suppose that $A: V \rightarrow V^{*}$ is both monotone and hemicontinuous with respect to the first argument of $N$ and $T: V \rightarrow V^{*}$ is continuous with respect to the second argument of $N$. Let $g: V \rightarrow V$ be continuous and $G: V \rightarrow \mathbf{R} \cup\{+\infty\}$ be a proper, convex, and lower semicontinuous functional. Then, for all $\epsilon>0, \Omega(\epsilon)=\Psi(\epsilon)$ is closed in $V$.

Theorem 3.10. Suppose that $A: V \rightarrow V^{*}$ is both monotone and hemicontinuous with respect to the first argument of $N$ and $T: V \rightarrow V^{*}$ is continuous with respect to the second argument of $N$. Let $g: V \rightarrow V$ be continuous and $G: V \rightarrow \mathbf{R} \cup\{+\infty\}$ a proper, convex, and lower semicontinuous functional. Then, the SMVHVI is strongly well posed if and only if

$$
\Omega(\epsilon) \neq \emptyset, \quad \forall \epsilon>0, \quad \operatorname{diam} \Omega(\epsilon) \longrightarrow 0 \quad \text { as } \epsilon \longrightarrow 0
$$

Proof. "Necessity". Suppose that the SMVHVI is strongly well posed. Then the SMVHVI has a unique solution which lies in $\Omega(\epsilon)$ and so $\Omega(\epsilon) \neq \emptyset$ for all $\epsilon>0$. If $\operatorname{diam} \Omega(\epsilon) \nrightarrow 0$ as $\epsilon \rightarrow 0$, then there exist a constant $l>0$, a nonnegative sequence $\left\{\epsilon_{n}\right\}$ with $\epsilon_{n} \rightarrow 0$ and $u_{n}, v_{n} \in \Omega\left(\epsilon_{n}\right)$ such that

$$
\left\|u_{n}-v_{n}\right\|_{V}>l, \quad \forall n \geq 1
$$

Since $u_{n}, v_{n} \in \Omega\left(\epsilon_{n}\right)$, it is known that $\left\{u_{n}\right\}$ and $\left\{v_{n}\right\}$ are both approximating sequences for the SMVHVI. From the strong well-posedness of the SMVHVI, it follows that both $\left\{u_{n}\right\}$ and $\left\{v_{n}\right\}$ converge strongly to the unique solution of the SMVHVI, which is a contradiction to (3.13). 
"Sufficiency". Let $\left\{u_{n}\right\} \subset V$ be an approximating sequence for the SMVHVI. Then there exists a nonnegative sequence $\left\{\epsilon_{n}\right\}$ with $\epsilon_{n} \rightarrow 0$ such that

$$
\begin{array}{r}
\left\langle N\left(A g\left(u_{n}\right), T u_{n}\right)-f, v-g\left(u_{n}\right)\right\rangle+J^{\circ}\left(u_{n}, v-g\left(u_{n}\right)\right)+G(v)-G\left(g\left(u_{n}\right)\right) \geq-\epsilon_{n}\left\|v-g\left(u_{n}\right)\right\|_{V^{\prime}} \\
\forall v \in V,
\end{array}
$$

which implies that $u_{n} \in \Omega\left(\epsilon_{n}\right)$. By condition (3.12), $\left\{u_{n}\right\}$ is a Cauchy sequence and so $\left\{u_{n}\right\}$ converges strongly to some point $u \in V$. Since the mapping $A$ is monotone with respect to the first argument of $N$, the mapping $T$ is continuous with respect to the second argument of $N, g$ is continuous, the Clarke's generalized directional derivative $J^{\circ}(u, v)$ is upper semicontinuous with respect to $(u, v)$, and $G$ is lower semicontinuous, it follows from (3.14) that

$$
\begin{aligned}
& \langle N(A v, T u)-f, v-g(u)\rangle+J^{\circ}(u, v-g(u))+G(v)-G(g(u)) \\
& \quad \geq \limsup _{n \rightarrow \infty}\left(\left\langle N\left(A v, T u_{n}\right)-f, v-g\left(u_{n}\right)\right\rangle+J^{\circ}\left(u_{n}, v-g\left(u_{n}\right)\right)+G(v)-G\left(g\left(u_{n}\right)\right)\right) \\
& \quad \geq \limsup _{n \rightarrow \infty}\left(\left\langle N\left(A g\left(u_{n}\right), T u_{n}\right)-f, v-g\left(u_{n}\right)\right\rangle+J^{\circ}\left(u_{n}, v-g\left(u_{n}\right)\right)+G(v)-G\left(g\left(u_{n}\right)\right)\right) \\
& \quad \geq \limsup _{n \rightarrow \infty}\left(-\epsilon_{n}\left\|v-g\left(u_{n}\right)\right\|_{V}\right) \\
& \quad=0, \quad \forall v \in V .
\end{aligned}
$$

Furthermore, since $A$ is also hemicontinuous with respect to the first argument of $N$ and $G$ is convex, by the argument similar to that in Lemma 3.7 we can readily prove that

$$
\langle N(A g(u), T u)-f, v-g(u)\rangle+J^{\circ}(u, v-g(u))+G(v)-G(g(u)) \geq 0, \quad \forall v \in V,
$$

which implies that $u$ solves the SMVHVI.

To complete the proof of Theorem 3.10, we need only to prove that the SMVHVI has a unique solution. Assume by contradiction that the SMVHVI has two distinct solutions $u_{1}$ and $u_{2}$. Then it is easy to see that $u_{1}, u_{2} \in \Omega(\epsilon)$ for all $\epsilon>0$ and

$$
0<\left\|u_{1}-u_{2}\right\|_{V} \leq \operatorname{diam} \Omega(\epsilon) \longrightarrow 0,
$$

which is a contradiction. Therefore, the SMVHVI has a unique solution. This completes the proof.

Corollary 3.11 (see [30, Theorem 3.1]). Suppose that $A: V \rightarrow V^{*}$ is a monotone and hemicontinuous mapping, $T: V \rightarrow V^{*}$ is a continuous mapping, and $G: V \rightarrow \mathbf{R} \cup\{+\infty\}$ is a proper, convex, and lower semicontinuous functional. Then, the VHVI is strongly well posed if and only if

$$
\Omega(\epsilon) \neq \emptyset, \quad \forall \epsilon>0, \quad \operatorname{diam} \Omega(\epsilon) \longrightarrow 0 \quad \text { as } \epsilon \longrightarrow 0 .
$$


Proof. In Theorem 3.10, put $N\left(u^{*}, v^{*}\right)=u^{*}+v^{*}$, for all $u^{*}, v^{*} \in V^{*}$ and $g=I$ the identity mapping of $V$. Then from the monotonicity and hemicontinuity of $A$ it follows that $A: V \rightarrow$ $V^{*}$ is both monotone and hemicontinuous with respect to the first argument of $N$. Moreover, from the continuity of $T$ it follows that $T: V \rightarrow V^{*}$ is continuous with respect to the second argument of $N$. Thus, utilizing Theorem 3.10, we obtain the desired result.

Theorem 3.12. Suppose that $A: V \rightarrow V^{*}$ is both monotone and hemicontinuous with respect to the first argument of $N$ and $T: V \rightarrow V^{*}$ is continuous with respect to the second argument of $N$. Let $g: V \rightarrow V$ be continuous and $G: V \rightarrow \mathbf{R} \cup\{+\infty\}$ be a proper, convex, and lower semicontinuous functional. Then, the SMVHVI is strongly well posed in the generalized sense if and only if

$$
\Omega(\epsilon) \neq \emptyset, \quad \forall \epsilon>0, \quad \mu(\Omega(\epsilon)) \longrightarrow 0 \quad \text { as } \epsilon \longrightarrow 0
$$

Proof. "Necessity". Suppose that the SMVHVI is strongly well posed in the generalized sense. Then the solution set of the SMVHVI is nonempty and $S \subset \Omega(\epsilon)$ for any $\epsilon>0$. Furthermore, the solution set of the SMVHVI also is compact. In fact, for any sequence $\left\{u_{n}\right\} \subset S$, it follows from $S \subset \Omega(\epsilon)$ for any $\epsilon>0$ that $\left\{u_{n}\right\} \subset S$ is an approximating sequence for the SMVHVI. Since the SMVHVI is strongly well posed in the generalized sense, $\left\{u_{n}\right\}$ has a subsequence which converges strongly to some point of the solution set $S$. Thus, the solution set $S$ of the SMVHVI is compact. Now let us show that $\mu(\Omega(\epsilon)) \rightarrow 0$ as $\epsilon \rightarrow 0$. From $S \subset \Omega(\epsilon)$ for any $\epsilon>0$, we get

$$
\mathscr{H}(\Omega(\epsilon), S)=\max \{e(\Omega(\epsilon), S), e(S, \Omega(\epsilon))\}=e(\Omega(\epsilon), S) .
$$

Taking into account the compactness of the solution set $S$, we obtain from (3.20) that

$$
\mu(\Omega(\epsilon)) \leq 2 \mathscr{\ell}(\Omega(\epsilon), S)=2 e(\Omega(\epsilon), S) .
$$

In order to prove that $\mu(\Omega(\epsilon)) \rightarrow 0$ as $\epsilon \rightarrow 0$, it is sufficient to show that $e(\Omega(\epsilon), S) \rightarrow 0$ as $\epsilon \rightarrow 0$. Assume by contradiction that $e(\Omega(\epsilon), S) \nrightarrow 0$ as $\epsilon \rightarrow 0$. Then there exist a constant $l>0$, a sequence $\left\{\epsilon_{n}\right\} \subset \mathbf{R}_{+}$with $\epsilon_{n} \rightarrow 0$ and $u_{n} \in \Omega\left(\epsilon_{n}\right)$ such that

$$
u_{n} \notin S+B(0, l),
$$

where $B(0, l)$ is the closed ball centered at 0 with radius $l$. Since $\left\{u_{n}\right\}$ is an approximating sequence for the SMVHVI and the SMVHVI is strongly well posed in the generalized sense, there exists a subsequence $\left\{u_{n_{k}}\right\}$ which converges strongly to some point $u \in S$ which is a contradiction to (3.22). Then $\mu(\Omega(\epsilon)) \rightarrow 0$ as $\epsilon \rightarrow 0$.

"Sufficiency". Assume that condition (3.19) holds. By Corollary 3.9, we conclude that $\Omega(\epsilon)$ is nonempty and closed for all $\epsilon>0$. Observe that

$$
S=\bigcap_{\epsilon>0} \Omega(\epsilon) .
$$


Since $\mu(\Omega(\epsilon)) \rightarrow 0$ as $\epsilon \rightarrow 0$, by applying the theorem [36, page 412], it can be easily found that $S$ is nonempty and compact with

$$
e(\Omega(\epsilon), S)=\mathscr{H}(\Omega(\epsilon), S) \longrightarrow 0 \quad \text { as } \epsilon \longrightarrow 0
$$

Let $\left\{u_{n}\right\} \subset V$ be an approximating sequence for the SMVHVI. Then there exists a nonnegative sequence $\left\{\epsilon_{n}\right\}$ with $\epsilon_{n} \rightarrow 0$ such that

$$
\begin{array}{r}
\left\langle N\left(A g\left(u_{n}\right), T u_{n}\right)-f, v-g\left(u_{n}\right)\right\rangle+J^{\circ}\left(u_{n}, v-g\left(u_{n}\right)\right)+G(v)-G\left(g\left(u_{n}\right)\right) \geq-\epsilon_{n}\left\|v-g\left(u_{n}\right)\right\|_{V^{\prime}} \\
\forall v \in V,
\end{array}
$$

and so $u_{n} \in \Omega\left(\epsilon_{n}\right)$ by the definition of $\Omega\left(\epsilon_{n}\right)$. It follows from (3.24) that

$$
d\left(u_{n}, S\right) \leq e(\Omega(\epsilon), S) \longrightarrow 0 .
$$

Since the solution set $S$ is compact, there exists $\bar{u}_{n} \in S$ such that

$$
\left\|u_{n}-\bar{u}_{n}\right\|_{V}=d\left(u_{n}, S\right) \longrightarrow 0
$$

Again from the compactness of the solution set $S,\left\{\bar{u}_{n}\right\}$ has a subsequence $\left\{\bar{u}_{n_{k}}\right\}$ converging strongly to some $\bar{u} \in S$. It follows from (3.27) that

$$
\left\|u_{n_{k}}-\bar{u}\right\|_{V} \leq\left\|u_{n_{k}}-\bar{u}_{n_{k}}\right\|_{V}+\left\|\bar{u}_{n_{k}}-\bar{u}\right\|_{V} \longrightarrow 0,
$$

which implies that $\left\{u_{n_{k}}\right\}$ converges strongly to $\bar{u}$. Therefore, the SMVHVI is strongly wellposed in the generalized sense. This completes the proof.

Corollary 3.13 (see [30, Theorem 3.2]). Suppose that $A: V \rightarrow V^{*}$ is a monotone and hemicontinuous mapping, $T: V \rightarrow V^{*}$ is a continuous mapping, and $G: V \rightarrow \mathbf{R} \cup\{+\infty\}$ is a proper, convex, and lower semicontinuous functional. Then, the VHVI is strongly well posed in the generalized sense if and only if

$$
\Omega(\epsilon) \neq \emptyset, \quad \forall \epsilon>0, \quad \mu(\Omega(\epsilon)) \longrightarrow 0 \quad \text { as } \epsilon \longrightarrow 0 .
$$

The following theorem gives some conditions under which the strongly mixed variational-hemivariational inequality is strongly well posed in the generalized sense in Euclidean space $\mathbf{R}^{n}$.

Theorem 3.14. Suppose that $A: \mathbf{R}^{n} \rightarrow \mathbf{R}^{n}$ is both monotone and hemicontinuous with respect to the first argument of $N$ and $T: \mathbf{R}^{n} \rightarrow \mathbf{R}^{n}$ is continuous with respect to the second argument of $N$. Let $g: \mathbf{R}^{n} \rightarrow \mathbf{R}^{n}$ be continuous and $G: \mathbf{R}^{n} \rightarrow \mathbf{R} \cup\{+\infty\}$ be a proper, convex, and lower semicontinuous functional. If there exists some $\epsilon>0$ such that $\Omega(\epsilon)$ is nonempty and bounded. Then the strongly mixed variational-hemivariational inequality SMVHVI is strongly well posed in the generalized sense. 
Proof. Suppose that $\left\{u_{n}\right\}$ is an approximating sequence for the SMVHVI. Then there exists a nonnegative sequence $\left\{\epsilon_{n}\right\}$ with $\epsilon_{n} \rightarrow 0$ as $n \rightarrow \infty$ such that

$$
\begin{array}{r}
\left\langle N\left(A g\left(u_{n}\right), T u_{n}\right)-f, v-g\left(u_{n}\right)\right\rangle+J^{\circ}\left(u_{n}, v-g\left(u_{n}\right)\right)+G(v)-G\left(g\left(u_{n}\right)\right) \geq-\epsilon_{n}\left\|v-g\left(u_{n}\right)\right\|_{\mathbf{R}^{n}} \\
\forall v \in \mathbf{R}^{n} .
\end{array}
$$

Let $\epsilon_{0}>0$ be such that $\Omega\left(\epsilon_{0}\right)$ is nonempty and bounded. Then there exists $n_{0}$ such that $u_{n} \in$ $\Omega\left(\epsilon_{0}\right)$ for all $n>n_{0}$. This implies that $\left\{u_{n}\right\}$ is bounded by the boundedness of $\Omega\left(\epsilon_{0}\right)$. Thus, there exists a subsequence $\left\{u_{n_{k}}\right\}$ such that $u_{n_{k}} \rightarrow \bar{u}$ as $k \rightarrow \infty$. Since the mapping $A$ is monotone with respect to the first argument of $N$, the mapping $T$ is continuous with respect to the second argument of $N, g$ is continuous, the Clarke's generalized directional derivative $J^{\circ}(u, v)$ is upper semicontinuous with respect to $(u, v)$, and $G$ is lower semicontinuous, it follows from (3.30) that

$$
\begin{aligned}
& \langle N(A v, T \bar{u})-f, v-g(\bar{u})\rangle+J^{\circ}(\bar{u}, v-g(\bar{u}))+G(v)-G(g(\bar{u})) \\
& \quad \geq \limsup _{k \rightarrow \infty}\left(\left\langle N\left(A v, T u_{n_{k}}\right)-f, v-g\left(u_{n_{k}}\right)\right\rangle+J^{\circ}\left(u_{n_{k}}, v-g\left(u_{n_{k}}\right)\right)+G(v)-G\left(g\left(u_{n_{k}}\right)\right)\right) \\
& \quad \geq \limsup _{k \rightarrow \infty}\left(\left\langle N\left(A g\left(u_{n_{k}}\right), T u_{n_{k}}\right)-f, v-g\left(u_{n_{k}}\right)\right\rangle+J^{\circ}\left(u_{n_{k}}, v-g\left(u_{n_{k}}\right)\right)+G(v)-G\left(g\left(u_{n_{k}}\right)\right)\right) \\
& \quad \geq \limsup _{k \rightarrow \infty}\left(-\epsilon_{n_{k}}\left\|v-g\left(u_{n_{k}}\right)\right\|_{\mathbf{R}^{n}}\right) \\
& \quad=0, \quad \forall v \in \mathbf{R}^{n} .
\end{aligned}
$$

Meantime, since $A$ is also hemicontinuous with respect to the first argument of $N$ and $G$ is convex, by the argument similar to that in Lemma 3.7 we can readily prove that

$$
\langle N(A g(\bar{u}), T \bar{u})-f, v-g(\bar{u})\rangle+J^{\circ}(\bar{u}, v-g(\bar{u}))+G(v)-G(g(\bar{u})) \geq 0, \quad \forall v \in \mathbf{R}^{n},
$$

which implies that $\bar{u}$ solves the SMVHVI. Therefore, the SMVHVI is strongly well-posed in the generalized sense. This completes the proof.

Corollary 3.15 (see [30, Theorem 3.3]). Suppose that $A: \mathbf{R}^{n} \rightarrow \mathbf{R}^{n}$ is a monotone and hemicontinuous mapping, $T: \mathbf{R}^{n} \rightarrow \mathbf{R}^{n}$ is a continuous mapping, and $G: \mathbf{R}^{n} \rightarrow \mathbf{R} \cup\{+\infty\}$ is a proper, convex, and lower semicontinuous functional. If there exists some $\epsilon>0$ such that $\Omega(\epsilon)$ is nonempty and bounded. Then the variational-hemivariational inequality VHVI is strongly well posed in the generalized sense.

\section{Well-Posedness of Inclusion Problem}

In this section, we first recall the concept of well-posedness for inclusion problems and then investigate the relations between the well-posedness for the strongly mixed variationalhemivariational inequality and the well-posedness for the corresponding inclusion problem. 
In what follows we always assume that $F$ is a set-valued mapping from real reflexive Banach space $V$ to its dual space $V^{*}$. The inclusion problem associated with mapping $F$ is defined by

$$
\operatorname{IP}(F): \quad \text { find } x \in V \text { such that } 0 \in F(x) \text {. }
$$

Definition 4.1 (see $[18,37]$ ). A sequence $\left\{u_{n}\right\} \subset V$ is called an approximating sequence for the inclusion problem $\operatorname{IP}(F)$ if $d\left(0, F\left(u_{n}\right)\right) \rightarrow 0$ or, equivalently, there exists a sequence $w_{n} \in$ $F\left(u_{n}\right)$ such that $\left\|w_{n}\right\|_{V^{*}} \rightarrow 0$ as $n \rightarrow \infty$.

Definition 4.2 (see $[18,37]$ ). We say that the inclusion problem $\operatorname{IP}(F)$ is strongly (resp., weakly) well posed if it has a unique solution and every approximating sequence converges strongly (resp., weakly) to the unique solution of IP $(F)$.

Definition 4.3 (see $[18,37]$ ). We say that the inclusion problem $\operatorname{IP}(F)$ is strongly (resp., weakly) well posed in the generalized sense if the solution set $S$ of the $\operatorname{IP}(F)$ is nonempty and every approximating sequence has a subsequence which converges strongly (resp., weakly) to some point of the solution set $S$ for the $\operatorname{IP}(F)$.

The following two theorems establish the relations between the strong (resp., weak) well-posedness for the strongly mixed variational-hemivariational inequality and the strong (resp., weak) well-posedness for the corresponding inclusion problem.

Theorem 4.4. Let $N: V^{*} \times V^{*} \rightarrow V^{*}, A, T: V \rightarrow V^{*}$, and $g: V \rightarrow V$ be four mappings, $J: V \rightarrow \mathbf{R}$ a locally Lipschitz functional, and $G: V \rightarrow \mathbf{R} \cup\{+\infty\}$ a proper, convex, and lower semicontinuous functional. Then the strongly mixed variational-hemivariational inequality $S M V H V I$ is strongly (resp., weakly) well posed if and only if the corresponding inclusion problem $\operatorname{IP}(N(A(g), T)-f+\bar{\partial} J+\partial G(g))$ is strongly (resp., weakly) well posed.

Theorem 4.5. Let $N: V^{*} \times V^{*} \rightarrow V^{*}, A, T: V \rightarrow V^{*}$, and $g: V \rightarrow V$ be four mappings, $J: V \rightarrow \mathbf{R}$ a locally Lipschitz functional, and $G: V \rightarrow \mathbf{R} \cup\{+\infty\}$ a proper, convex, and lower semicontinuous functional. Then the strongly mixed variational-hemivariational inequality SMVHVI is strongly (resp., weakly) well posed in the generalized sense if and only if the corresponding inclusion problem $\operatorname{IP}(N(A(g), T)-f+\bar{\partial} J+\partial G(g))$ is strongly (resp., weakly) well posed in the generalized sense.

Lemma 4.6. Let $N: V^{*} \times V^{*} \rightarrow V^{*}, A, T: V \rightarrow V^{*}$, and $g: V \rightarrow V$ be four mappings, $J: V \rightarrow \mathbf{R}$ a locally Lipschitz functional, and $G: V \rightarrow \mathbf{R} \cup\{+\infty\}$ a proper, convex, and lower semicontinuous functional. Then $u \in V$ is a solution of the SMVHVI if and only if $u$ is a solution of the corresponding inclusion problem $\operatorname{IP}(N(A(g), T)-f+\bar{\partial} J+\partial G(g))$ of finding $u \in V$ such that

$$
0 \in N(A(g(u)), T u)-f+\bar{\partial} J(u)+\partial G(g(u))
$$

Proof. "Sufficiency". Assume that $u$ is a solution of the inclusion problem $\operatorname{IP}(N(A(g), T)-$ $f+\bar{\partial} J+\partial G(g))$. Then there exist $w_{1} \in \bar{\partial} J(u)$ and $w_{2} \in \partial G(g(u))$ such that

$$
N(A(g(u)), T u)-f+w_{1}+w_{2}=0 .
$$


By multiplying $v-g(u)$ at both sides of the above equation (4.3), we obtain from the definitions of the Clarke's generalized gradient for locally Lipschitz functional and the subgradient for convex functional that

$$
\begin{aligned}
0 & =\left\langle N(A(g(u)), T u)-f+w_{1}+w_{2}, v-g(u)\right\rangle \\
& \leq\langle N(A(g(u)), T u)-f, v-g(u)\rangle+J^{\circ}(u, v-g(u))+G(v)-G(g(u)), \quad \forall v \in V,
\end{aligned}
$$

which implies that $u$ is a solution of the SMVHVI.

"Necessity". Suppose that $u$ is a solution of the SMVHVI. Then,

$$
\langle N(A(g(u)), T u)-f, v-g(u)\rangle+J^{\circ}(u, v-g(u))+G(v)-G(g(u)) \geq 0, \quad \forall v \in V .
$$

From the fact that

$$
J^{\circ}(u, v-g(u))=\max \{\langle w, v-g(u)\rangle: w \in \bar{\partial} J(u)\},
$$

we deduce that there exists a $w(u, v) \in \bar{\partial} J(u)$ such that

$$
\langle N(A(g(u)), T u)-f, v-g(u)\rangle+\langle w(g(u), v), v-g(u)\rangle+G(v)-G(g(u)) \geq 0, \quad \forall v \in V .
$$

In terms of Proposition 2.4 (iv), $\bar{\partial} J(u)$ is a nonempty, convex, bounded, weak*-compact subset of $V^{*}$. Note that $V$ is a real reflexive Banach space. Hence, $\bar{\partial} J(u)$ is a nonempty, convex, bounded, weak-compact subset in $V^{*}$. Thus $\bar{\partial} J(u)$ is a nonempty, closed, convex, and bounded subset in $V^{*}$ which implies that $\{N(A(g(u)), T u)-f+w: w \in \bar{\partial} J(u)\}$ is nonempty, closed, convex, and bounded in $V^{*}$. Since $G: V \rightarrow \mathbf{R} \cup\{+\infty\}$ is a proper, convex, and lower semicontinuous functional, it follows from Theorem 2.7 with $\varphi(u)=G(u)$ and (4.7) that there exists $w(u) \in \bar{\partial} J(u)$ such that

$$
\langle N(A(g(u)), T u)-f, v-g(u)\rangle+\langle w(u), v-g(u)\rangle+G(v)-G(g(u)) \geq 0, \quad \forall v \in V .
$$

For the sake of simplicity we write $w=w(u)$, and hence from (4.8) we have

$$
G(v)-G(g(u)) \geq\langle-N(A(g(u)), T u)+f-w, v-g(u)\rangle, \quad \forall v \in V,
$$

which implies that $-N(A(g(u)), T u)+f-w \in \partial G(g(u))$. Consequently, it follows from $w \in$ $\bar{\partial} J(u)$ that

$$
0 \in N(A(g(u)), T u)-f+\bar{\partial} J(u)+\partial G(g(u))
$$

which implies that $u$ is a solution of the inclusion problem $\operatorname{IP}(N(A(g), T)-f+\bar{\partial} J+\partial G(g))$. This completes the proof. 
Proof of Theorem 4.4. "Necessity". Assume that the SMVHVI is strongly (resp., weakly) well posed. Then there is a unique solution $u^{*}$ for the SMVHVI. By Lemma 4.6, $u^{*}$ also is the unique solution for the inclusion problem $\operatorname{IP}(N(A(g), T)-f+\bar{\partial} J+\partial G(g))$. Let $\left\{u_{n}\right\}$ be an approximating sequence for the $\operatorname{IP}(N(A(g), T)-f+\bar{\partial} J+\partial G(g))$. Then there exists a sequence $w_{n} \in N\left(A\left(g\left(u_{n}\right)\right), T u_{n}\right)-f+\bar{\partial} J\left(u_{n}\right)+\partial G\left(g\left(u_{n}\right)\right)$ such that $\left\|w_{n}\right\|_{V^{*}} \rightarrow 0$ as $n \rightarrow \infty$. And so there exist $\xi_{n} \in \bar{\partial} J\left(u_{n}\right)$ and $\eta_{n} \in \partial G\left(g\left(u_{n}\right)\right)$ such that

$$
w_{n}=N\left(A\left(g\left(u_{n}\right)\right), T u_{n}\right)-f+\xi_{n}+\eta_{n} .
$$

From the definitions of the Clarke's generalized gradient for locally Lipschitz functional and the subgradient for convex functional, we obtain by multiplying $v-g\left(u_{n}\right)$ at both sides of the above equation (4.11) that

$$
\begin{aligned}
\langle N( & \left.\left.A\left(g\left(u_{n}\right)\right), T u_{n}\right)-f, v-g\left(u_{n}\right)\right\rangle+J^{\circ}\left(u_{n}, v-g\left(u_{n}\right)\right)+G(v)-G\left(g\left(u_{n}\right)\right) \\
& \geq\left\langle N\left(A\left(g\left(u_{n}\right)\right), T u_{n}\right)-f, v-g\left(u_{n}\right)\right\rangle+\left\langle\xi_{n}, v-g\left(u_{n}\right)\right\rangle+\left\langle\eta_{n}, v-g\left(u_{n}\right)\right\rangle \\
& =\left\langle w_{n}, v-g\left(u_{n}\right)\right\rangle \\
& \geq-\left\|w_{n}\right\|_{V^{*}}\left\|v-g\left(u_{n}\right)\right\|_{V^{\prime}} \quad \forall v \in V .
\end{aligned}
$$

Letting $\epsilon_{n}=\left\|w_{n}\right\|_{V^{*}}$, we obtain that $\left\{u_{n}\right\}$ is an approximating sequence for the SMVHVI from (4.12) with $\left\|w_{n}\right\|_{V^{*}} \rightarrow 0$ as $n \rightarrow \infty$. Therefore, it follows from the strong (resp., weak) wellposedness of the SMVHVI that $\left\{u_{n}\right\}$ converges strongly (resp., weakly) to the unique solution $u^{*}$. Thus, the inclusion problem $\operatorname{IP}(N(A(g), T)-f+\bar{\partial} J+\partial G(g))$ is strongly (resp., weakly) well posed.

"Sufficiency". Suppose that the inclusion problem $\operatorname{IP}(N(A(g), T)-f+\bar{\partial} J+\partial G(g))$ is strongly (resp., weakly) well posed. Then the $\operatorname{IP}(N(A(g), T)-f+\bar{\partial} J+\partial G(g))$ has a unique solution $u^{*}$, which implies that $u^{*}$ is the unique solution for the SMVHVI by Lemma 4.6. Let $\left\{u_{n}\right\}$ be an approximating sequence for the SMVHVI. Then there exists a sequence $\left\{\epsilon_{n}\right\}$ with $\epsilon_{n} \rightarrow 0$ as $n \rightarrow \infty$ such that

$$
\begin{array}{r}
\left\langle N\left(A\left(g\left(u_{n}\right)\right), T u_{n}\right)-f, v-g\left(u_{n}\right)\right\rangle+J^{\circ}\left(u_{n}, v-g\left(u_{n}\right)\right)+G(v)-G\left(g\left(u_{n}\right)\right) \geq-\epsilon_{n}\left\|v-g\left(u_{n}\right)\right\|_{V^{\prime}} \\
\forall v \in V .
\end{array}
$$

By the same argument as in the proof of Lemma 4.6, there exists a $w\left(u_{n}, v\right) \in \bar{\partial} J\left(u_{n}\right)$ such that

$$
\begin{aligned}
& \left\langle N\left(A\left(g\left(u_{n}\right)\right), T u_{n}\right)-f, v-g\left(u_{n}\right)\right\rangle+\left\langle w\left(g\left(u_{n}\right), v\right), v-g\left(u_{n}\right)\right\rangle+G(v)-G\left(g\left(u_{n}\right)\right) \\
& \quad \geq-\epsilon_{n}\left\|v-g\left(u_{n}\right)\right\|_{V}, \quad \forall v \in V,
\end{aligned}
$$


and $\left\{N\left(A\left(g\left(u_{n}\right)\right), T u_{n}\right)-f+w: w \in \bar{\partial} J\left(u_{n}\right)\right\}$ is nonempty, closed, convex, and bounded in $V^{*}$. Then, it follows from (4.14) and Theorem 2.7 with $\varphi(u)=G(u)+\epsilon_{n}\left\|u-g\left(u_{n}\right)\right\|_{V}$, which is proper, convex, and lower semicontinuous, that there exists $w\left(u_{n}\right) \in \bar{\partial} J\left(u_{n}\right)$ such that

$$
\begin{aligned}
& \left\langle N\left(A\left(g\left(u_{n}\right)\right), T u_{n}\right)-f, v-g\left(u_{n}\right)\right\rangle+\left\langle w\left(u_{n}\right), v-g\left(u_{n}\right)\right\rangle+G(v)-G\left(g\left(u_{n}\right)\right) \\
& \quad \geq-\epsilon_{n}\left\|v-g\left(u_{n}\right)\right\|_{V^{\prime}}, \quad \forall v \in V .
\end{aligned}
$$

For the sake of simplicity we write $w_{n}=w\left(u_{n}\right)$, and hence from (4.15) we have

$$
G\left(g\left(u_{n}\right)\right) \leq G(v)+\left\langle N\left(A\left(g\left(u_{n}\right)\right), T u_{n}\right)-f+w_{n}, v-g\left(u_{n}\right)\right\rangle+\epsilon_{n}\left\|v-g\left(u_{n}\right)\right\|_{V^{\prime}} \quad \forall v \in V .
$$

Define functional $\bar{G}_{n}: V \rightarrow \mathbf{R} \cup\{+\infty\}$ as follows:

$$
\bar{G}_{n}(v)=G(v)+P_{n}(v)+\epsilon_{n} Q_{n}(v),
$$

where $P_{n}(v)$ and $Q_{n}(v)$ are two functionals on $V$ defined by

$$
P_{n}(v)=\left\langle N\left(A\left(g\left(u_{n}\right)\right), T u_{n}\right)-f+w_{n}, v-g\left(u_{n}\right)\right\rangle, \quad Q_{n}(v)=\left\|v-g\left(u_{n}\right)\right\|_{V} .
$$

Clearly, $\bar{G}_{n}$ is proper, convex, and lower semicontinuous and $v=g\left(u_{n}\right)$ is a global minimizer of $\bar{G}_{n}$ on $V$. Thus, $0 \in \partial \bar{G}_{n}\left(g\left(u_{n}\right)\right)$. Since the functionals $P_{n}$ and $Q_{n}$ are continuous on $V$ and $G$ is proper, convex, and lower semicontinuous, it follows from Proposition 2.3 that

$$
\partial \bar{G}_{n}(v)=\partial G(v)+N\left(A\left(g\left(u_{n}\right)\right), T u_{n}\right)-f+w_{n}+\epsilon_{n} \partial Q_{n}(v) .
$$

It is easy to calculate that

$$
\partial Q_{n}(v)=\left\{v^{*} \in V^{*}:\left\|v^{*}\right\|_{V^{*}}=1,\left\langle v^{*}, v-g\left(u_{n}\right)\right\rangle=\left\|v-g\left(u_{n}\right)\right\|_{V}\right\},
$$

and so there exists a $\xi_{n} \in \partial Q_{n}\left(g\left(u_{n}\right)\right)$ with $\left\|\xi_{n}\right\|_{V^{*}}=1$ such that

$$
0 \in \partial G\left(g\left(u_{n}\right)\right)+N\left(A\left(g\left(u_{n}\right)\right), T u_{n}\right)-f+w_{n}+\epsilon_{n} \xi_{n} .
$$

Letting $u_{n}^{*}=-\epsilon_{n} \xi_{n}$, we have $\left\|u_{n}^{*}\right\|_{V^{*}} \rightarrow 0$ as $\epsilon_{n} \rightarrow 0$. Moreover, since $w_{n} \in \bar{\partial} J\left(u_{n}\right)$, it follows from (4.21) that

$$
u_{n}^{*} \in N\left(A\left(g\left(u_{n}\right)\right), T u_{n}\right)-f+\bar{\partial} J\left(u_{n}\right)+\partial G\left(g\left(u_{n}\right)\right),
$$

which implies that $\left\{u_{n}\right\}$ is an approximating sequence for the $\operatorname{IP}(N(A(g), T)-f+\bar{\partial} J+\partial G(g))$. Since the inclusion problem $\operatorname{IP}(N(A(g), T)-f+\bar{\partial} J+\partial G(g))$ is strongly (resp., weakly) 
well posed, $\left\{u_{n}\right\}$ converges strongly (resp., weakly) to the unique solution $u^{*}$. Therefore, the strongly mixed variational-hemivariational inequality SMVHVI is strongly (resp., weakly) well posed. This completes the proof.

Proof of Theorem 4.5. The proof is similar to that in Theorem 4.4 and so we omit it here.

Corollary 4.7 (see [30, Theorem 4.1]). Let $A$ and $T$ be two mappings from Banach space $V$ to its dual $V^{*}, J: V \rightarrow \mathbf{R}$ be a locally Lipschitz functional, and $G: V \rightarrow \mathbf{R} \cup\{+\infty\}$ be a proper, convex, and lower semicontinuous functional. Then the variational-hemivariational inequality VHVI is strongly (resp., weakly) well posed if and only if the corresponding inclusion problem $\operatorname{IP}(A+T-f+\bar{\partial} J+\partial G)$ is strongly (resp., weakly) well posed.

Proof. In Theorem 4.4, put $g=I$ the identity mapping of $V$ and $N\left(u^{*}, v^{*}\right)=u^{*}+v^{*}$, for all $u^{*}, v^{*} \in V^{*}$. Then, in terms of Theorem 4.4 we derive the desired result.

Corollary 4.8 ([30, Theorem 4.2]). Let $A$ and $T$ be two mappings from Banach space $V$ to its dual $V^{*}, J: V \rightarrow \mathbf{R}$ be a locally Lipschitz functional, and $G: V \rightarrow \mathbf{R} \cup\{+\infty\}$ be a proper, convex, and lower semicontinuous functional. Then the variational-hemivariational inequality VHVI is strongly (resp., weakly) well posed in the generalized sense if and only if the corresponding inclusion problem $\mathrm{IP}(A+T-f+\bar{\partial} J+\partial G)$ is strongly (resp., weakly) well posed in the generalized sense.

\section{Concluding Remarks}

In this paper, we introduce some concepts of well-posedness for a class of strongly mixed variational-hemivariational inequalities with perturbations, which includes as a special case the class of variational-hemivariational inequalities in [30]. We establish some metric characterizations for the well-posed strongly mixed variational-hemivariational inequality and give some conditions under which the strongly mixed variational-hemivariational inequality is strongly well posed in the generalized sense in $\mathbf{R}^{n}$. On the other hand, we first recall the concept of well-posedness for inclusion problems and then investigate the relations between the strong (resp., weak) well-posedness for a strongly mixed variational-hemivariational inequality and the strong (resp., weak) well-posedness for the corresponding inclusion problem.

It is well known that there are many other concepts of well-posedness for optimization problems, variational inequalities, and Nash equilibrium problems, such as $\alpha$-well-posedness [17], well-posedness by perturbations [12], and Levitin-Polyak well-posedness [38]. However, we wonder whether the concepts mentioned as above can be extended to the strongly mixed variational-hemivariational inequality. Beyond question, this is an interesting problem.

\section{Acknowledgments}

This paper was partially supported by the National Science Foundation of China (11071169), Innovation Program of Shanghai Municipal Education Commission (09ZZ133), and Leading Academic Discipline Project of Shanghai Normal University (DZL707) (to L.-C. Ceng). This paper was partially supported by the Taiwan NSC Grant 99-2115-M-110-007-MY3 (to N.-C. Wang). This paper was partially supported by the Taiwan NSC Grant 99-2221-E-037-007-MY3 (to J.-C. Yao). 


\section{References}

[1] A. N. Tykhonov, "Stability of a problem of optimization of functionals," Akademija Nauk SSSR, vol. 6, pp. 631-634, 1966.

[2] G. P. Crespi, A. Guerraggio, and M. Rocca, "Well posedness in vector optimization problems and vector variational inequalities," Journal of Optimization Theory and Applications, vol. 132, no. 1, pp. 213-226, 2007.

[3] A. L. Dontchev and T. Zolezzi, Well-Posed Optimization Problems, vol. 1543, Springer, Berlin, Germany, 1993.

[4] X.X. Huang, "Extended and strongly extended well-posedness of set-valued optimization problems," Mathematical Methods of Operations Research, vol. 53, no. 1, pp. 101-116, 2001.

[5] R. Lucchetti and F. Patrone, "A characterization of Tyhonov well-posedness for minimum problems, with applications to variational inequalities," Numerical Functional Analysis and Optimization, vol. 3, no. 4, pp. 461-476, 1981.

[6] T. Zolezzi, "Extended well-posedness of optimization problems," Journal of Optimization Theory and Applications, vol. 91, no. 1, pp. 257-266, 1996.

[7] Y.-P. Fang and R. Hu, "Parametric well-posedness for variational inequalities defined by bifunctions," Computers \& Mathematics with Applications, vol. 53, no. 8, pp. 1306-1316, 2007.

[8] Y.-P. Fang, R. Hu, and N.-J. Huang, "Well-posedness for equilibrium problems and for optimization problems with equilibrium constraints," Computers $\mathcal{E}$ Mathematics with Applications, vol. 55, no. 1, pp. 89-100, 2008.

[9] L. C. Ceng, N. Hadjisavvas, S. Schaible, and J. C. Yao, “Well-posedness for mixed quasivariational-like inequalities," Journal of Optimization Theory and Applications, vol. 139, no. 1, pp. 109-125, 2008.

[10] L. C. Ceng and J. C. Yao, "Well-posedness of generalized mixed variational inequalities, inclusion problems and fixed-point problems," Nonlinear Analysis. Theory, Methods E Applications, vol. 69, no. 12, pp. 4585-4603, 2008.

[11] Y.-P. Fang, N.-J. Huang, and J.-C. Yao, "Well-posedness of mixed variational inequalities, inclusion problems and fixed point problems," Journal of Global Optimization, vol. 41, no. 1, pp. 117-133, 2008.

[12] Y.-P. Fang, N.-J. Huang, and J.-C. Yao, "Well-posedness by perturbations of mixed variational inequalities in Banach spaces," European Journal of Operational Research, vol. 201, no. 3, pp. 682-692, 2010.

[13] M. B. Lignola, "Well-posedness and L-well-posedness for quasivariational inequalities," Journal of Optimization Theory and Applications, vol. 128, no. 1, pp. 119-138, 2006.

[14] D. Goeleven and D. Mentagui, "Well-posed hemivariational inequalities," Numerical Functional Analysis and Optimization, vol. 16, no. 7-8, pp. 909-921, 1995.

[15] E. Cavazzuti and J. Morgan, "Well-posed saddle point problems," in Optimization: Theory and Algorithms, vol. 86, pp. 61-76, Marcel Dekker, New York, Ny, USA, 1983.

[16] L. Q. Anh, P. Q. Khanh, D. T. M. Van, and J.-C. Yao, "Well-posedness for vector quasiequilibria," Taiwanese Journal of Mathematics, vol. 13, no. 2, pp. 713-737, 2009.

[17] X.-J. Long and N.-J. Huang, "Metric characterizations of $\alpha$-well-posedness for symmetric quasiequilibrium problems," Journal of Global Optimization, vol. 45, no. 3, pp. 459-471, 2009.

[18] B. Lemaire, C. Ould Ahmed Salem, and J. P. Revalski, "Well-posedness by perturbations of variational problems," Journal of Optimization Theory and Applications, vol. 115, no. 2, pp. 345-368, 2002.

[19] P. D. Panagiotopoulos, "Nonconvex energy functions. Hemivariational inequalities and substationarity principles," Acta Mechanica, vol. 48, no. 3-4, pp. 111-130, 1983.

[20] F. H. Clarke, Optimization and Nonsmooth Analysis, vol. 5, SIAM, Philadelphia, Pa, USA, 2nd edition, 1990.

[21] D. Motreanu and P. D. Panagiotopoulos, Minimax Theorems and Qualitative Properties of the Solutions of Hemivariational Inequalities, vol. 29, Kluwer Academic Publishers, Dordrecht, The Netherlands, 1999.

[22] P. D. Panagiotopoulos, Hemivariational Inequalities, Springer, Berlin, Germany, 1993.

[23] S. Carl, V. K. Le, and D. Motreanu, "Evolutionary variational-hemivariational inequalities: existence and comparison results," Journal of Mathematical Analysis and Applications, vol. 345, no. 1, pp. 545-558, 2008.

[24] S. Carl and D. Motreanu, "Extremal solutions of quasilinear parabolic inclusions with generalized Clarke's gradient," Journal of Differential Equations, vol. 191, no. 1, pp. 206-233, 2003.

[25] Z. H. Liu, "Existence results for quasilinear parabolic hemivariational inequalities," Journal of Differential Equations, vol. 244, no. 6, pp. 1395-1409, 2008.

[26] Z. H. Liu and D. Motreanu, "A class of variational-hemivariational inequalities of elliptic type," Nonlinearity, vol. 23, no. 7, pp. 1741-1752, 2010. 
[27] Y.-B. Xiao and N.-J. Huang, "Sub-supersolution method and extremal solutions for higher order quasi-linear elliptic hemi-variational inequalities," Nonlinear Analysis. Theory, Methods E Applications, vol. 66, no. 8, pp. 1739-1752, 2007.

[28] Y.-B. Xiao and N.-J. Huang, "Generalized quasi-variational-like hemivariational inequalities," Nonlinear Analysis. Theory, Methods \& Applications, vol. 69, no. 2, pp. 637-646, 2008.

[29] Y.-B. Xiao and N.-J. Huang, "Sub-super-solution method for a class of higher order evolution hemivariational inequalities," Nonlinear Analysis. Theory, Methods \& Applications, vol. 71, no. 1-2, pp. 558$570,2009$.

[30] Y. B. Xiao and N. J. Huang, "Well-posedness for a class of variational-hemivariational inequalities with perturbations," Journal of Optimization Theory and Applications, vol. 151, no. 1, pp. 33-51, 2011.

[31] F.-Q. Xia, N.-J. Huang, and Z.-B. Liu, "A projected subgradient method for solving generalized mixed variational inequalities," Operations Research Letters, vol. 36, no. 5, pp. 637-642, 2008.

[32] R. T. Rockafellar, Convex Analysis, Princeton University Press, Princeton, NJ, USA, 1997.

[33] S. Carl, V. K. Le, and D. Motreanu, Nonsmooth Variational Problems and Their Inequalities, Springer, New York, NY, USA, 2007.

[34] E. Zeidler, Nonlinear Functional Analysis and Its Applications, vol. 2, Springer, Berlin, Germany, 1990.

[35] F. Giannessi and A. A. Khan, "Regularization of non-coercive quasi variational inequalities," Control and Cybernetics, vol. 29, no. 1, pp. 91-110, 2000.

[36] K. Kuratowski, Topology, vol. 1-2, Academic Press, New York, NY, USA, 1968.

[37] B. Lemaire, "Well-posedness, conditioning, and regularization of minimization, inclusion, and fixed point problems," Pliska Studia Mathematica Bulgaria, vol. 12, pp. 71-84, 1998.

[38] X. X. Huang, X. Q. Yang, and D. L. Zhu, "Levitin-Polyak well-posedness of variational inequality problems with functional constraints," Journal of Global Optimization, vol. 44, no. 2, pp. 159-174, 2009. 


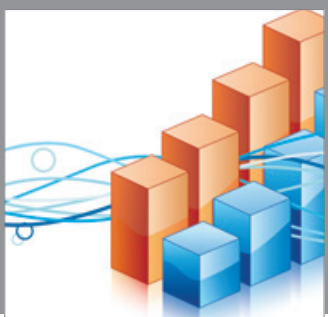

Advances in

Operations Research

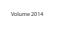

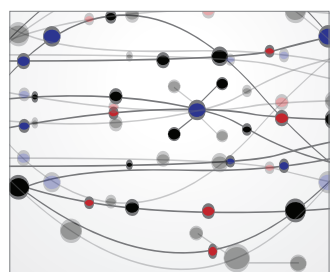

\section{The Scientific} World Journal
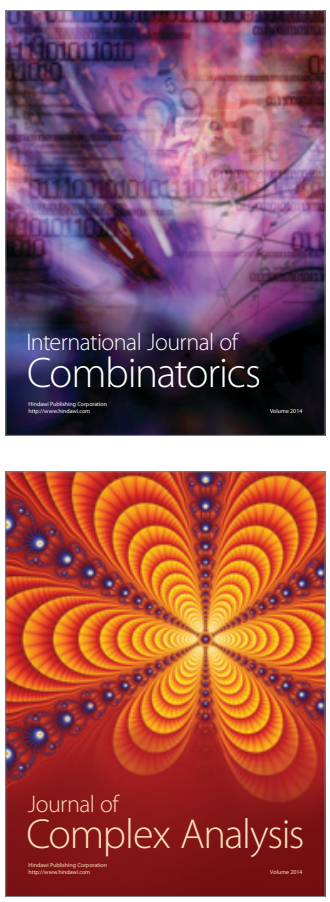

International Journal of

Mathematics and

Mathematical

Sciences
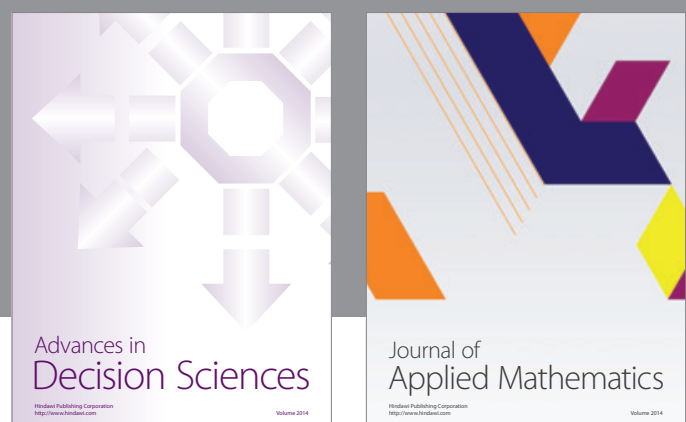

Journal of

Applied Mathematics
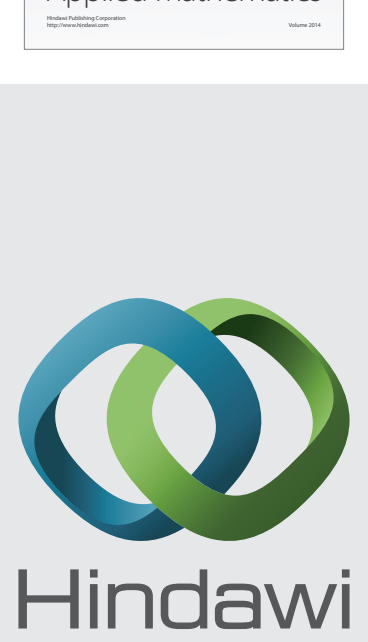

Submit your manuscripts at http://www.hindawi.com
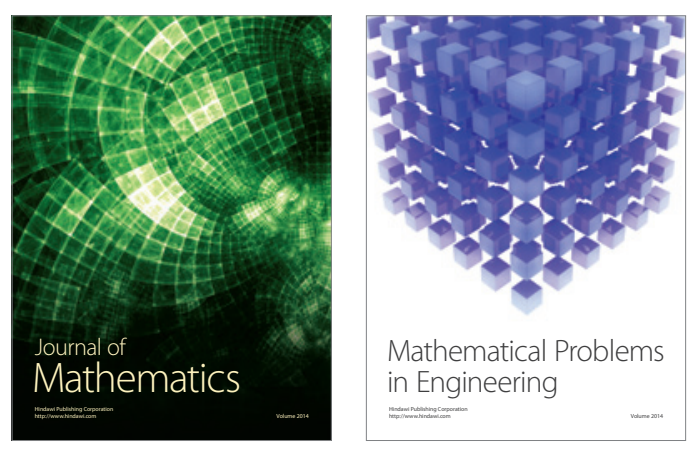

Mathematical Problems in Engineering
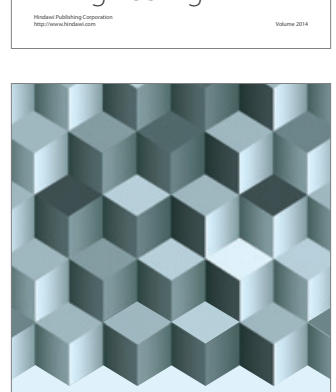

Journal of

Function Spaces
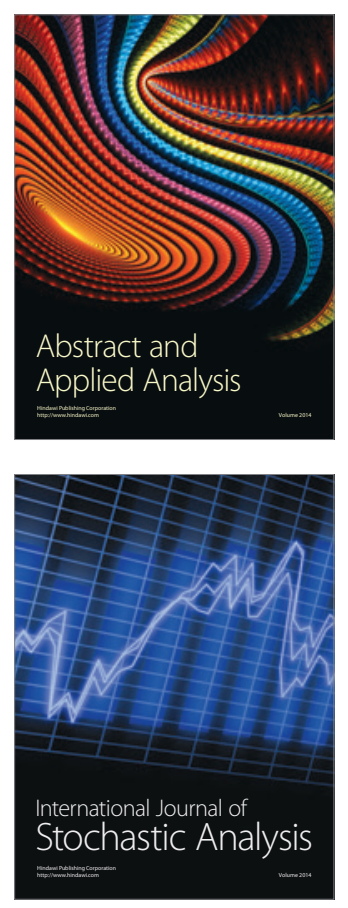

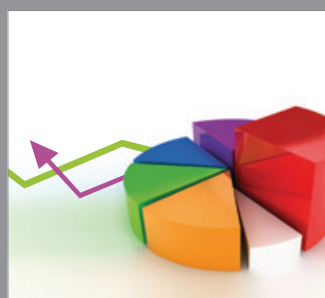

ournal of

Probability and Statistics

Promensencen
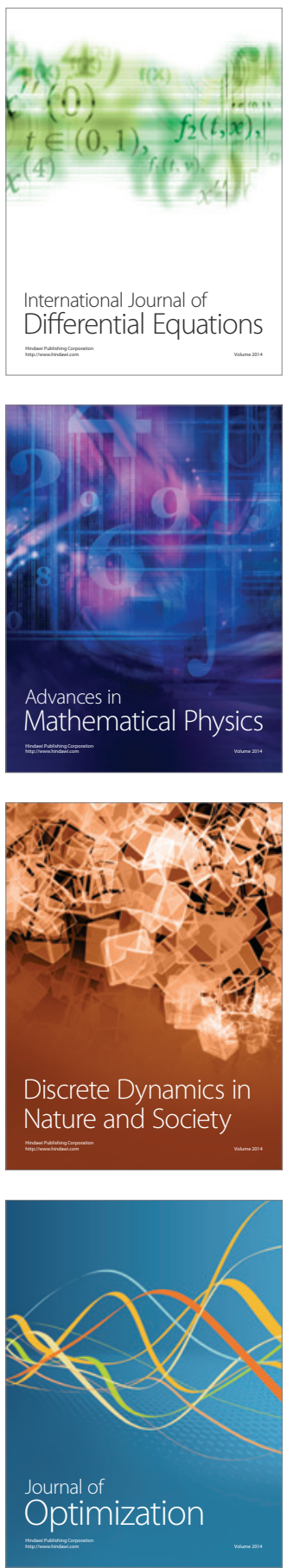